Original Research Paper

\title{
Impact of the Edges of a Backside Diffusive Reflector on the External Quantum Efficiency of Luminescent Solar Concentrators: Experimental and Computational Approach
}

\author{
${ }^{1}$ Mauro Pravettoni, ${ }^{1}$ Cristina S. Polo Lòpez and ${ }^{2}$ Robert P. Kenny \\ ${ }^{1}$ Institute of Applied Sustainability to the Built Environment, \\ University of Applied Sciences and Arts of Southern Switzerland (SUPSI), Canobbio, Switzerland \\ ${ }^{2}$ Institute for Energy and Transport, DG Joint Research Centre, European Commission, Ispra, Italy
}

Article history

Received: 13-01-2016

Revised: 15-01-2016

Accepted: 15-01-2016

Corresponding Author:

Mauro Pravettoni

Institute of Applied Sustainability

to the Built Environment,

University of Applied Sciences and Arts of Southern Switzerland (SUPSI), Canobbio, Switzerland Email: mauro.pravettoni@supsi.ch

\begin{abstract}
Luminescent Solar Concentrators (LSCs) have been proposed in the 1970s as cheap planar concentrators for residential applications and nowadays represent a novel idea with excellent perspectives for building integration photovoltaics. The interest in LSCs has increased in the last years, due to improved stability of luminescent dyes, the introduction of quantum dots and nanorods and the overall reported increase in module efficiency. Computational methods have been suddenly applied as an important tool for the description of light dynamics in LSCs. With "raytracing methods" light is described as particle-like (photons) and each particle is tracked. It is precious tool for the description of absorption/reemission events, refraction and internal reflection in LSCs. It is also a very useful approach for the description of LSC edge effects, which may be well described by means of basic geometrical optics and are the subject of this work. The impact of scattering layers on the backside of LSCs is analysed in detail both experimentally and computationally. Results give evidence of the non-wavelength dependent impact of backside diffusers to the external quantum efficiency of LSCs and thus to their overall performance. A possible design of LSC as smart windows in photovoltaic facades is also suggested, where the benefits of the edge effects described are taken into account.
\end{abstract}

Keywords: Photovoltaics, Luminescent Solar Concentrators, Monte Carlo Modelling

\section{Introduction}

Luminescent Solar Concentrators (LSCs) are static planar photovoltaic (PV) concentrators and have been proposed by Weber and Lambe (1976) and by Goetzberger and Gruebel (1977). In LSCs a refractive material (typically glass or PMMA matrix) is filled with fluorescent particles, either dyes or quantum dots (Barnham et al., 2000), that absorb photons at certain wavelengths and re-emit them at higher wavelengths. A fraction of re-emitted light (here simply referred to as luminescent light) is transmitted to the PV cell at edge of the concentrator by total internal reflection, with a gain factor $G$ given by the ratio between the PV cell area and the receiver area. In principle, the spectral range of the luminescent light can be controlled by selecting appropriate luminescent species and the efficiency may be enhanced by reflectors at the rear surface.

The interest in LSCs has increased in the last years, due to improved stability of luminescent dyes (Rowan et al., 2008), the introduction of quantum dots and nanorods (Bose et al., 2008) and the overall reported increase in module efficiency (Slooff et al., 2006; Currie et al., 2008); Goldschmidt et al., 2009). Debije and Verbunt (2011), Farrell and Yoshida (2012) and previously Van Sark et al. (2008) described the most recent results in LCS development and characterization. 


\section{LSC as a Concentrating Photovoltaic Building Integration Resource}

Conventional concentrating photovoltaic (CPV) systems require one or two axis solar tracking to collect the direct light. Under highly concentrated light, the PV cell reaches also high temperatures and active cooling is required to avoid performance degradation. Both these aspects, in addition to a bulkier module compared to traditional nonconcentrating flat panel PV modules, are particularly challenging in view of the integration of $\mathrm{CPV}$ into buildings and as a consequence CPV has found applications only in large solar farms to date.

Today, residential construction is called to make a major contribution to reducing energy waste, environmental sustainability and comfort. New standards are required by the industry to develop more efficient buildings. Among these, the European Directive 31 of 2010 that will impose for 2020 to all buildings constructed in Europe to self-produce through all the renewable energy needed to cover consumption towards net zero energy buildings. To achieve these goals in the near future, it will be extremely helpful to use not only the roofs to integrate PV systems but also facades.

Solar transmission through window glazing affects not only the air-conditioning load into a building, but also the thermal and visual comfort. Glazing with advanced thermal and optical properties for energy conservation and aesthetic purposes, such as low emissivity glass and switchable glazing, are already commercially available. The most common in buildingintegrated PV are the so-called "see-through" and "lightthrough" solar systems. Silicon solar cells and thin film solar cells are used today for semitransparent glasses for windows. Semi transparency in traditional building integrated PV can create a pleasant environment allowing natural light to enter the building.

Heat dissipation is another crucial problem. The houses are often built with materials which do not allow the heat containment and therefore require a great deal of energy to maintain a correct internal environmental condition.

Lighting and thermal environment comfort within these areas using PV glass and its incidence in the global energy building consumption is still under investigation (Polo Lopez et al., 2012). It is not known yet what effect transparent solar building integrated PV systems may cause to the sensory and psychological perception of people.

LSC panels represent a novel idea to get reasonable efficiencies with no-tracking CPV, with excellent prospects for cost reduction. In this study investigation in the LSC design is presented to help increasing performance, while maintaining good architectural integration perspectives as smart windows. LSCs collect diffuse light more efficiently than any other PV technology (Pravettoni et al., 2009a) and the module efficiency can be increased with backside diffusive reflectors.

The LCS systems are also typically transparent to the infrared radiation, thus avoiding the PV cell overheating. By contrast LCS elements are coloured according to the luminescent dye in use: this aspect makes LSCs especially attractive from an aesthetic point of view but should be studied to determine the impact that various colours have on the human mind.

\section{Computational Methods and Pre-Normative Electrical Characterization of LSCS}

Computational methods have been applied as an important tool for the description of light dynamics in LSCs since the 1980s (Carrascosa et al., 1983). There are basically two different approaches:

The thermodynamic approach (Yablonovitch, 1980; Chatten et al., 2005), based on the detailed balance principle and describing the radiative energy transfer between mesh points in the concentrator plate

The "ray-tracing method", in which light is described as particle-like (photons) and each particle is tracked. Ray-tracing method is usually a Monte Carlo code, where a random number generator is used to set the photons in their configurational phase-space. The ray-tracing approach is important for the description of absorption/re-emission events, refraction and internal reflection in LSCs. It is also a very useful tool for the description of LSC edge effects (Sidrach de Cardona et al., 1985; Pravettoni et al., 2008).

LSCs represent a challenge for the optical and electrical characterization, as has been early discussed by Batchelder et al. $(1979 ; 1981)$ and Soti et al. (1995). The impact of scattering layers has been analysed by Debije et al. (2009). In this study the authors show the impact on the External Quantum Efficiency (EQE) of a diffusive reflector (here simply referred to as diffusers) on the rear side of the LSC. The existing standard for SR measurements of flatpanel PV is considered as a guide to the characterization. Several configurations for the background diffuser are considered. Measurements are performed by means of dedicated experimental setups in two laboratories: The European Solar Test Installation (ESTI), a European centre of reference for standard measurements in PV where the world record efficient LSC to date was measured (Green et al., 2016); and the Experimental Solid State (EXSS) group laboratory for quantum efficiency measurements of the Blackett Laboratory at the Imperial College London (ICL), UK.

Experimental results are compared with the computational ones from Monte Carlo ray tracing simulations: As a result, the importance of light diffusion at the edges of the LSC is highlighted. This 
result gives new strength towards the development of LSCs as promising building integration PV elements for the years to come.

\section{Experimental Setup}

The procedure for Spectral Response (SR) measurements of flat-panel PV devices (cells or modules) is described by the international standard IEC 60904-8: the $S R$ (in $\mathrm{A} \mathrm{W}^{-1}$ ) is defined as the following ratio:

$$
S R(\lambda)=\frac{\delta J_{s c}}{E(\lambda) d \lambda}
$$

where, $\delta J_{s c}$ (in $\mathrm{A} \mathrm{m}^{-2}$ ) is the photogenerated shortcircuit current density from the test device, $E(\lambda)$ is the incident spectral irradiance (in $\mathrm{W} \mathrm{m}^{-2} \mathrm{~nm}^{-1}$ ) and $E(\lambda) d \lambda$ (in $\mathrm{W} \mathrm{m}{ }^{-2}$ ) is the total incident irradiance between $\lambda-\mathrm{d} \lambda / 2$ and $\lambda+\mathrm{d} \lambda / 2$.

$E(\lambda) d \lambda$ is detected by a reference cell of known $S R$; $\delta J_{s c}$ is measured from the test device.

If $N_{e}$ is the total number of electron-hole pairs produced and flowing into the external circuit and $N_{\lambda}$ is the total number of incident photons at wavelength $\lambda$ then the EQE can be defined and calculated as follows:

$$
\operatorname{EQE}(\lambda)=\frac{N_{e}}{N_{\lambda}}=\frac{h c}{q \lambda} S R(\lambda)
$$

where, $h=6.626 \times 10^{-34} \mathrm{~J}$ s is Planck's constant, $c=$ $2.998 \times 10^{8} \mathrm{~m} \mathrm{~s}^{-1}$ is the speed of light and $q=1.602 \times 10^{-19}$ $\mathrm{C}$ is the elementary charge. EQE is often used instead of $S R$ in the literature, meaning the probability that an incident photon at wavelength $\lambda$ will deliver an electron- hole pair to the external circuit which is collected at the contacts.

\section{Setup at the European Solar Test Installation}

The experimental setup in use at ESTI has been presented already in the literature (Pravettoni et al., 2009a). A $300 \mathrm{~W}$ steady-state xenon lamp provides the source light that is filtered by up to 80 bandpass filters ( 8 to $20 \mathrm{~nm}$ band width; wavelength range: 300 to 1650 $\mathrm{nm})$. The monochromatic light obtained is chopped, superimposed to a continuous bias light background and measured by a digital lock-in amplifier $(1 \mathrm{mHz}$ to 102.4 $\mathrm{kHz}$ frequency range, $2 \mathrm{nV}$ to $1 \mathrm{~V}$ sensitivity). Bias light is used in order to keep the test device under irradiance conditions close to the standard operating conditions.

Monochromatic light $\pm 5 \%$ uniformity up to $30 \times 30$ $\mathrm{cm}^{2}$ target area is provided by a lens system and allows simultaneous measurement of a small-size test device and of a c-Si ESTI reference cell of known absolute SR. The test device and the reference cell are usually kept at $(25 \pm 2){ }^{\circ} \mathrm{C}$ temperature by a cooling plate: PV cell in LSCs cannot be cooled by direct contact with the cooling plate but has been monitored with a Pt100 sensor at the backside of the PV cell, showing no significant temperature increase during the measurements.

The short-circuit current value $\delta J_{s c}$ in Equation 1 is measured over a calibrated shunt resistor. The resistance value is set in order to maintain the test device close to short-circuit. Measurement uncertainties associated with the expetimental setup described has been discussed in detail by Mullejans et al. (2009).

\section{Setup at Blackett Laboratory}

Figure 1 shows a picture of the experimental setup in use at the EXSS group at ICL for SR measurements of PV devices.

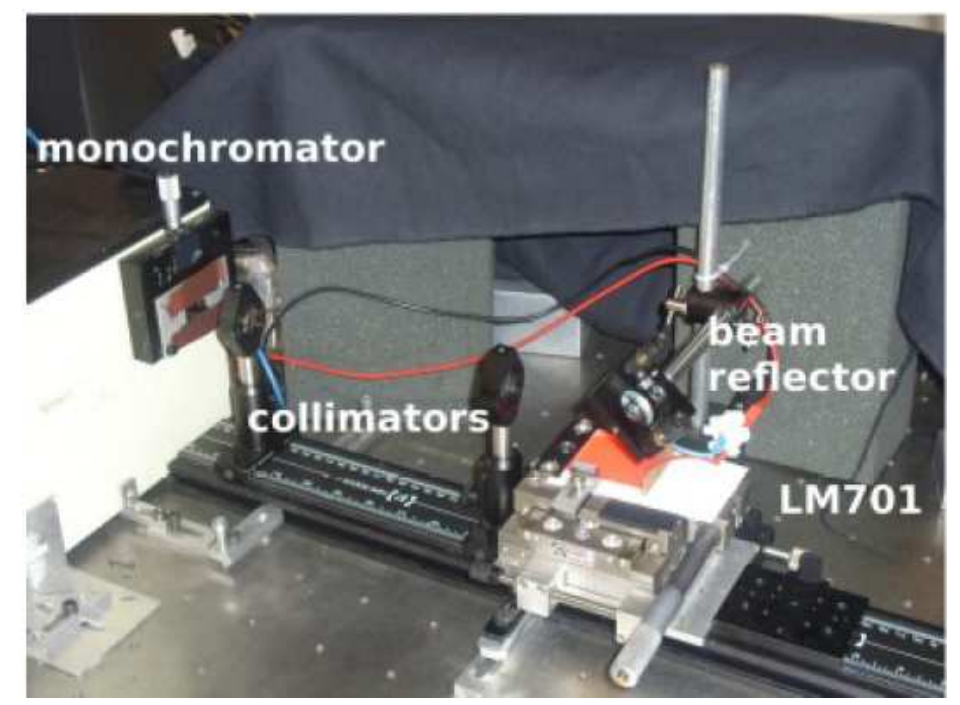

Fig. 1. SR measurement setup at the QE laboratory (EXSS group, Blackett Laboratory, ICL) 
Here the monochromatic light is produced by a monochromator, chopped and collimated onto the test device. In order to drive the pointlike monochromatic beam to the test $\mathrm{LSC}$, a $90^{\circ}$ beam reflector is used. The test LSC can be moved on a mechanical XY stage with micrometric control.

No bias light is used: Lock-in technique reduces the signal-to-noise ratio. A calibrated c-Si reference cell is used to detect the monochromatic beam intensity as above. No simultaneous measurement of the test device and of the reference cell is possible: Temporal stability of the monochromatic source is periodically checked. Since no bias light is used, which is the main responsible of the test device heating, temperature control is almost unnecessary and is not performed at ICL.

With respect of ESTI's setup described above, light can here be directed with high precision to several points of the receiver and the impact of the backside diffusive reflector can be analysed in detail.

\section{Results}

Table 1 lists two LSC modules that have been tested and characterized. Both devices have been assembled by ECN, the independent research institute for renewable energy of the Netherlands, in cooperation with the Fraunhofer Institute of Applied Polymer Research (Fraunhofer-IAP), a German research centre pioneering studies in polymer materials. One (LM701) or four (LM704) $5 \times 50 \mathrm{~mm}^{2}$ PV cells are placed on the edges of $5 \times 5 \times 0.5 \mathrm{~cm}^{3}$ Poly(Methyl Methacrylate) (PMMA) matrices. Mirror tapes by $3 \mathrm{M}$ (97\% reflectivity) are placed on the free edges of LM701. Cells are connected with index matched PE 399 KrystalFlex film.

Fluorescent dyes in use are indicated and specified in Table 2. Lumogen F Red305 is a perylene colour by BASF; yellow CRS040 is a coumarin by Radiant Color. In LM704 PV cells may be connected either in series or in parallel.

At the rear side of the collector, diffusive reflectors of different materials, size and shape have been tested. In all the measurements reported, the backside diffuser is a sheet of white paper of different covering area and shapes, placed over a black cloth used as absorber. The diffuser is not glued to the LSC backside: A thin air gap is therefore assumed between the LSC and the diffuser. Figure $2 \mathrm{a}-\mathrm{d}$ show the four configurations tested on LM701: The backside diffuser covers the rear side of the LSC only partially, near the edge where the GaAs cell is placed.

LM704 has been tested with the various configurations shown in Fig. 2e-h. Similarly to the LM701 case, different diffuser areas have been employed: In this case the diffuser is a frame of different thickness.

Results of EQE measurements on LM701 are shown in Fig. 3a. The chart highlights the two absorption peaks at $440 \mathrm{~nm}$ (coumarin) and at $578 \mathrm{~nm}$ (perylene).

Luminescent emission cuts off at $630 \mathrm{~nm}$ : The shoulder of non-zero EQE between 600 and $900 \mathrm{~nm}$ arises from the unabsorbed light, diffused at the matrixto-air interfaces into the GaAs cell, which has its bandgap cutoff at $900 \mathrm{~nm}$.

The larger overall EQE has been measured with the full diffuser covering the entire backside surface, while the smaller EQE when no diffuser is used, as expected.

Similar results are shown in Fig. 3c (LM704).

The different response to the presence of different diffusers on LM701 and LM704 is highlighted in Fig. $3 \mathrm{~b}$ and Fig. 3d, where the normalized EQE is plotted as a function of the fraction of the diffuser area coverage, at four different wavelengths: At 450 and $550 \mathrm{~nm}$ (near the peaks of luminescent absorptions), at $483 \mathrm{~nm}$ (between the peaks) and at $850 \mathrm{~nm}$ (where noluminescence occurs). As a result, the EQE at wavelengths where luminescence occurs is shown to increase linearly with the fraction of area coverage. At $850 \mathrm{~nm}$, the EQE increase is sharp and, especially in LM704, nearly constant when any diffuser is present, while significantly decreases when no diffuser is used (65\% EQE increase at $850 \mathrm{~nm}$ on LM704 between the "no diffuser" and the "full area diffuser" case; less than $5 \%$ only increase at peak of irradiances).

Table 1. Test devices (Slooff et al., 2006)

\begin{tabular}{lllllll}
\hline ESTI label & Dim $\left(\mathrm{cm}^{3}\right)$ & Matrix & Dye & Cell type & No. cell s & Gain $G$ \\
\hline LM701 & $5 \times 5 \times 0.5$ & PMMA & Yellow CRS 040 $(0.003 \%)$ & GaAs & 1 & 10.0 \\
& & & Lumogen F Red305 $(0.01 \%)$ & & \\
LM704 & $5 \times 5 \times 0.5$ & PMMA & Yellow CRS 040 $(0.003 \%)$ & GaA s & 4 & 2.5 \\
& & & Lumogen F Red305 $(0.01 \%)$ & & \\
\hline
\end{tabular}

Table 2. Luminescent dyes in use: Nominal values given in the cited references

\begin{tabular}{|c|c|c|c|c|}
\hline \multirow[b]{2}{*}{ Fluorescent dye } & \multirow[b]{2}{*}{ Chemical } & \multirow[b]{2}{*}{ Producer } & \multicolumn{2}{|l|}{ Peak (nm) } \\
\hline & & & Absorption & Emission \\
\hline Lumogen F Red 305 & Perylene & BASF & 578 & 613 \\
\hline Yellow CRS 040* & Coumarin & Radiant Color & 440 & 506 \\
\hline
\end{tabular}

*Later on renamed Yellow CFS 002 


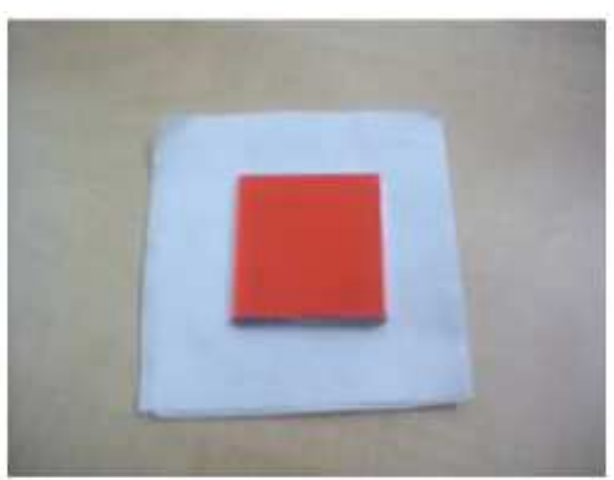

(a)

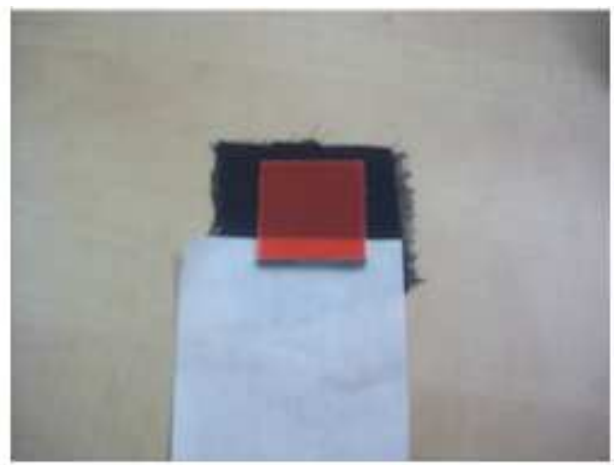

(c)

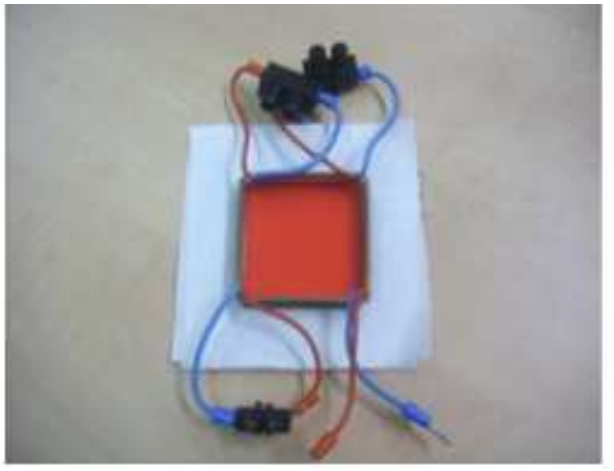

(e)

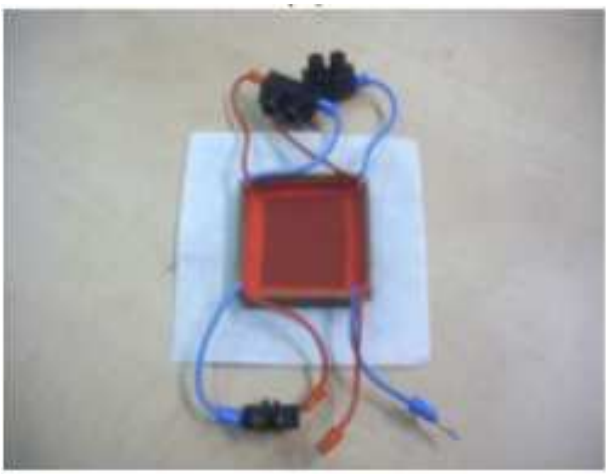

(f)

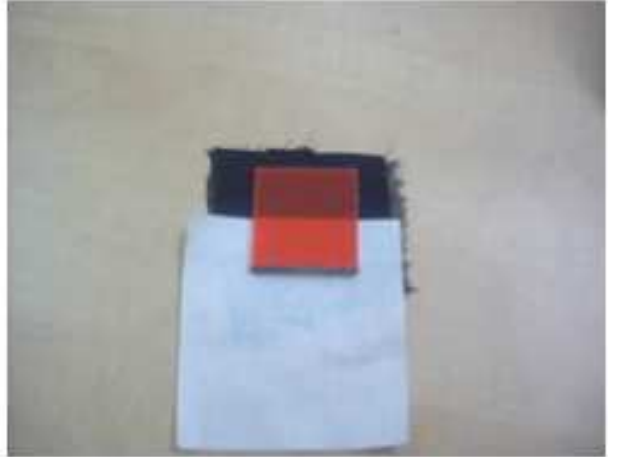

(b)

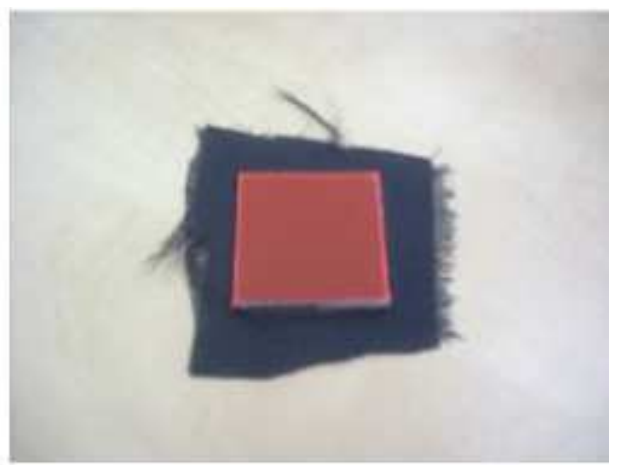

(d)

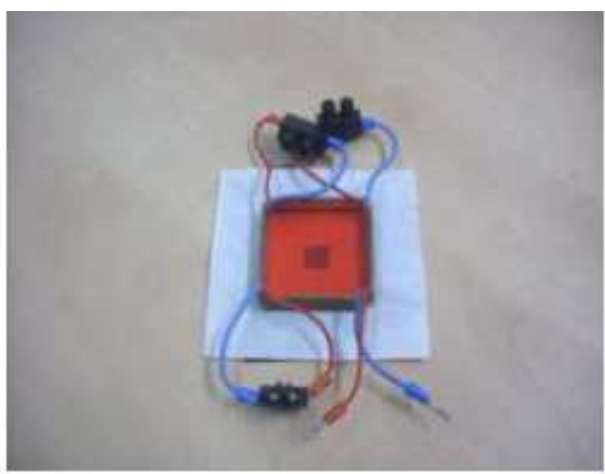

(f)

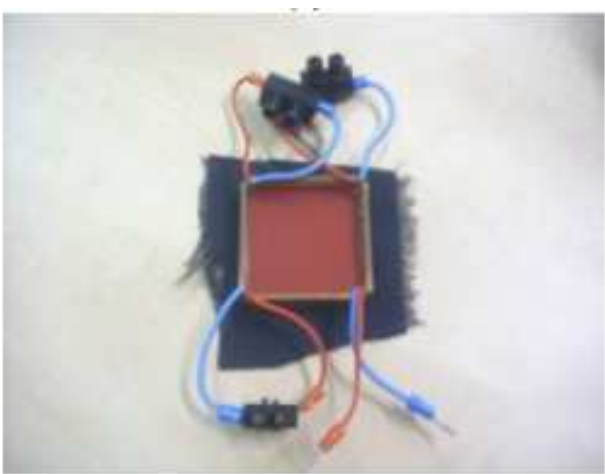

(h)

Fig. 2. Test devices with various backside configurations: (a-d) LM701 and (e-h) LM704 


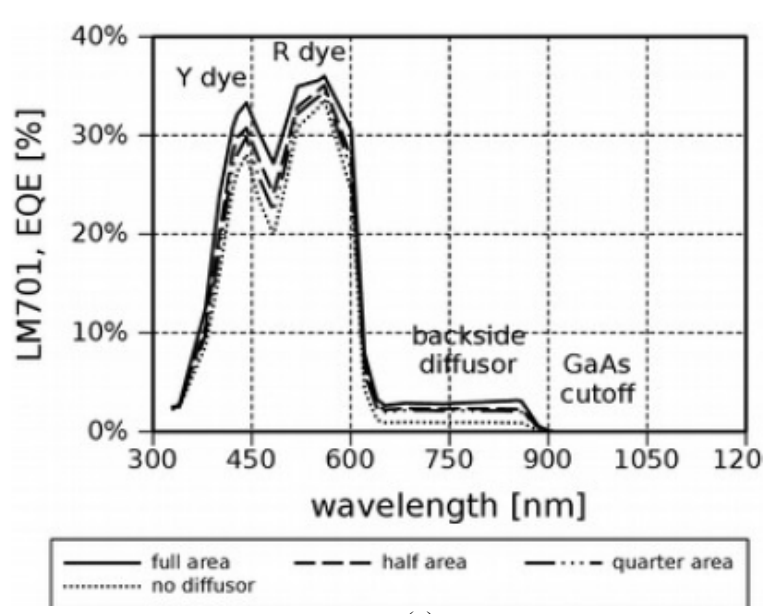

(a)

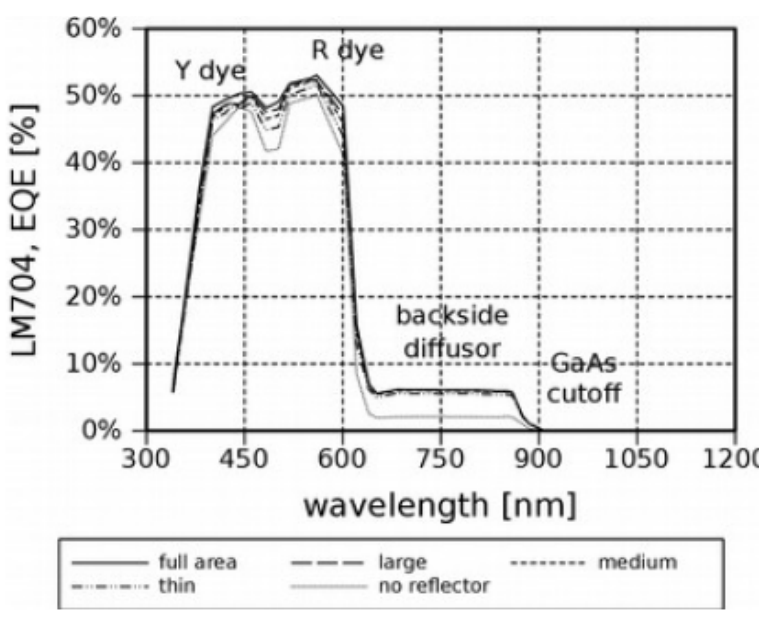

(c)

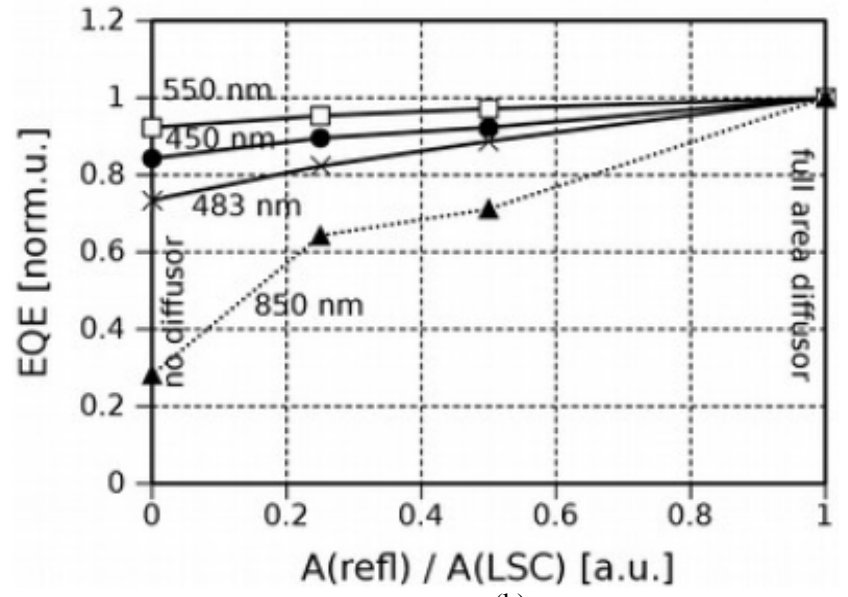

(b)

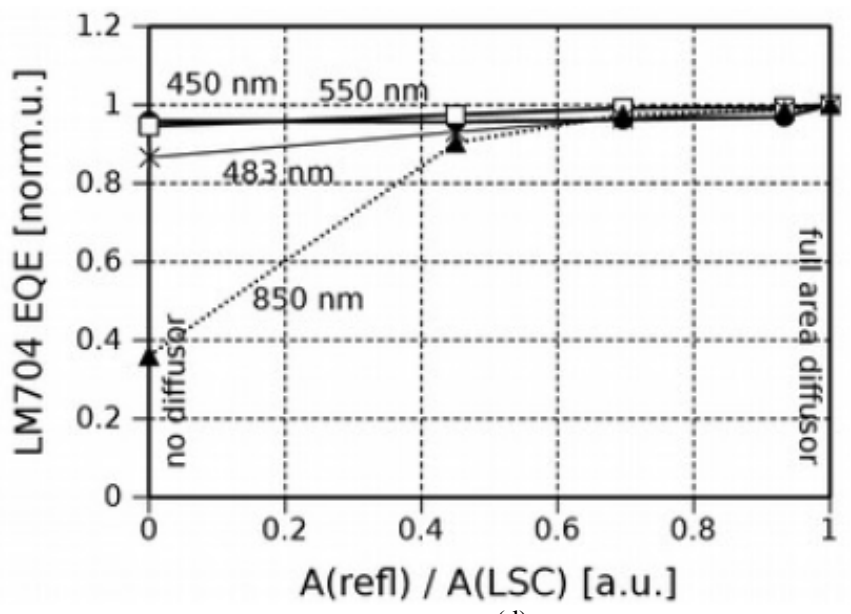

(d)

Fig. 3. EQE results at ESTI. (a) LM701 as a function of wavelength; (b) LM701 a function of reflector area. (c) LM704 as a function of wavelength; (d) LM704 as a function of reflector area

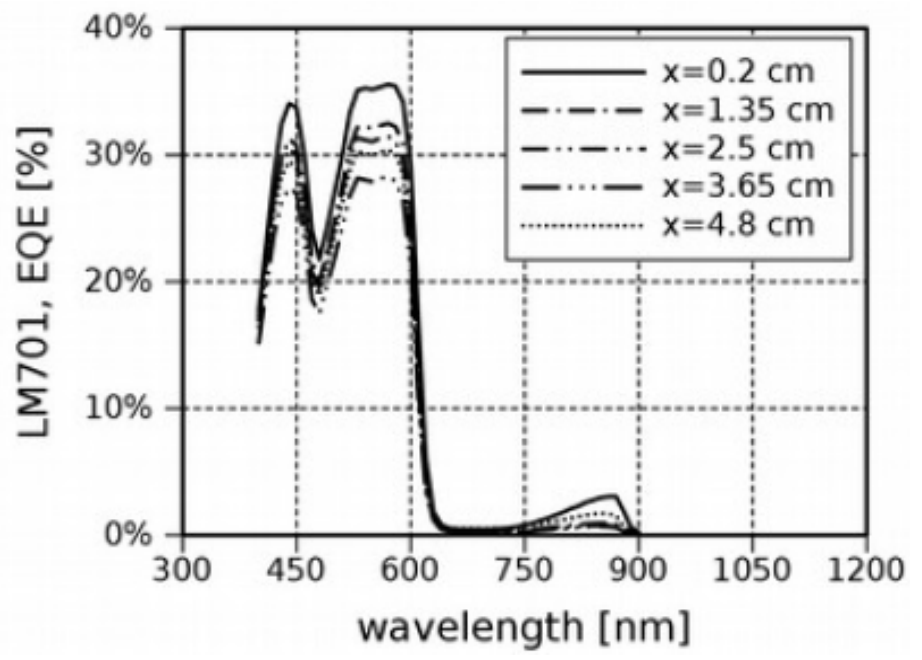

Fig. 4. EQE results at Blackett Laboratory: LM701 as a function of wavelength 


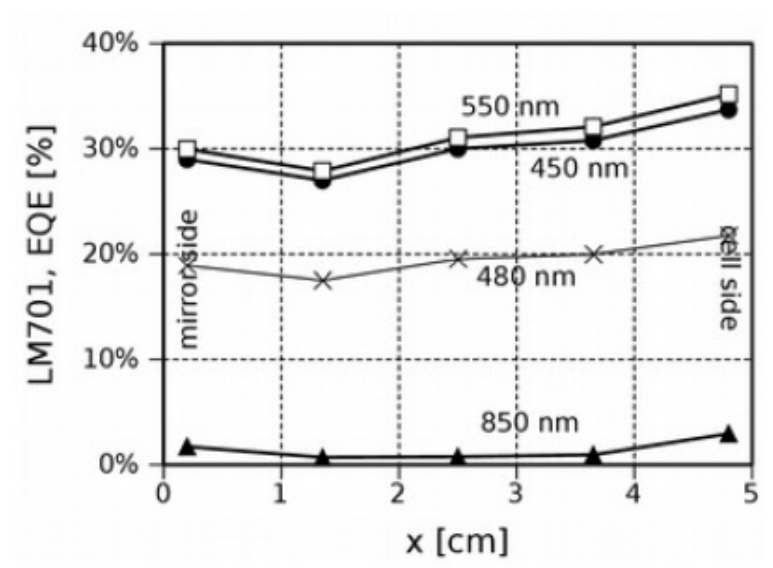

(a)

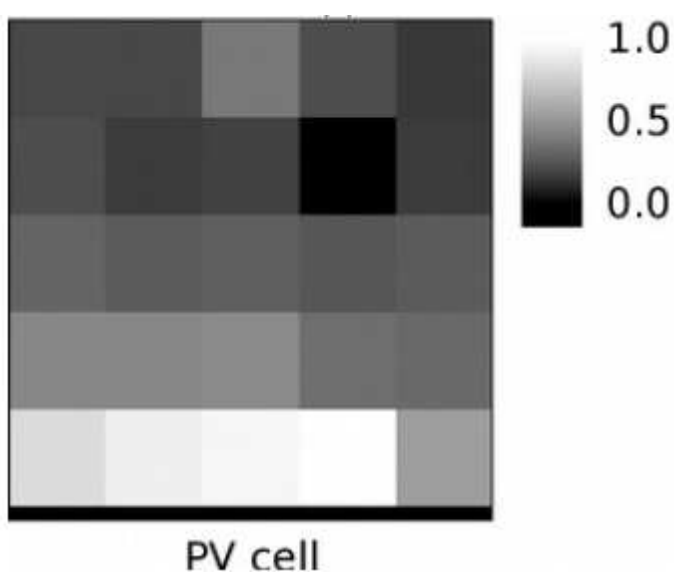

(b)

Fig. 5. EQE results at Blackett Laboratory: (a) As a function of the position, at various wavelengths; (b) Percentage distribution of EQE over LM701 receiving area

Figure 4 shows results of EQE measurements of LM701 on the experimental setup at ICL described above. EQE measurements have been performed in 25 points, equally distributed on a $5 \times 5$ square matrix. A white backside diffuser has been placed on the rear side of the LSC. Figure 5a shows the measured average EQE over the five measurements on a single row at position $x$ (mirror: $x=0 \mathrm{~cm}$; GaAs cell: $x=5$ $\mathrm{cm})$ and as a function of $x$.

Figure $5 \mathrm{~b}$ shows on a grayscale colormap, the impact of each of the 25 test positions to the overall integrated $\mathrm{EQE}$ (white is where local integrated EQE is maximum, black where it is minimum). All charts show that monochromatic light between $x=1$ and $x=2 \mathrm{~cm}$ and in the middle of the LSC gives less contribution to the global EQE, while a bigger contribution is given in the LSC region closest to the PV cell.

\section{The Effect of the Aperture Area}

In Equation 1 it is important to define the appropriate aperture area of the LSC module in the calculation of $\delta J_{s c}$. Several different area values can be considered for the LSCs listed in Table 1, whether or not the thin glue index matched layer connecting the PMMA matrix to the PV cells and the cells themselves are taken into account (Fig. 6a). Accurate area measurements have been performed at ESTI and are reported in the caption of Fig. 6.

In conventional flat panel modules, the area definition usually affects only the module efficiency calculation, since the area between cells is typically not active and does not contribute to the output power. However it may play an important role in certain devices, where it is proven that light diffuses from the inactive area between cells to the cells themselves. This is definitely the case of LSCs, where the glue layer diffuse light to the PV cell and the cell itself might intercept direct light other than the one emitted by luminescent dyes.

As a result, when the device is characterized it is important to define the aperture area, eventually through the application of a mask. Figure $6 \mathrm{~b}$ shows three different current-voltage characteristic curves at $1000 \mathrm{~W} \mathrm{~m}^{-2}$ and the calculated efficiencies on LM704, with no backside diffuser and with the three possible area definitions specified in the caption of Fig. 6a: (a) PMMA matrix only, giving $4.9 \%$ module efficiency; (b) including the thin glue layer, 5.1\%; and (c) including cell thickness, 5.2\%.

\section{Computational Model}

To better focus on the results in section above, the impact of a backside diffusive reflector was investigated with a monochromatic, non-wavelength dependent Monte Carlo ray-tracing code based on classical optics.

\section{Description}

In the ray-tracing approach, luminescent light is modelled by means of a set of $N$ monochromatic, unpolarized, non-interacting particles in a rectangular box with the dimensions of the testing LSCs. At start, particles randomly move in the matrix; when reaching the matrixto- air interfaces, particles undergo the laws of classical optics: Total internal reflection or refraction. Fresnel reflection is also taken into account: Particles in the escape cone can be reflected according to their reflectance which is calculated from the reflection coefficients in the unpolarized approximation. Secondary absorption is neglected.

Figure 7a shows the choice of coordinate systems: The origin in set in the middle of an edge (typically where a PV cell is located); the $z$-axis is along the edge main axis; $y$-axis has the same direction and versus of incoming light and the $\mathrm{x}$-axis forms a right-handed coordinate system. 


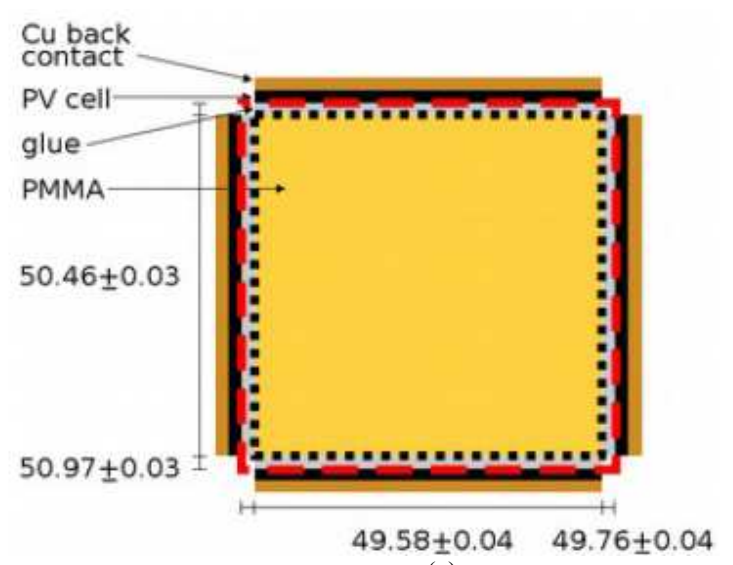

(a)

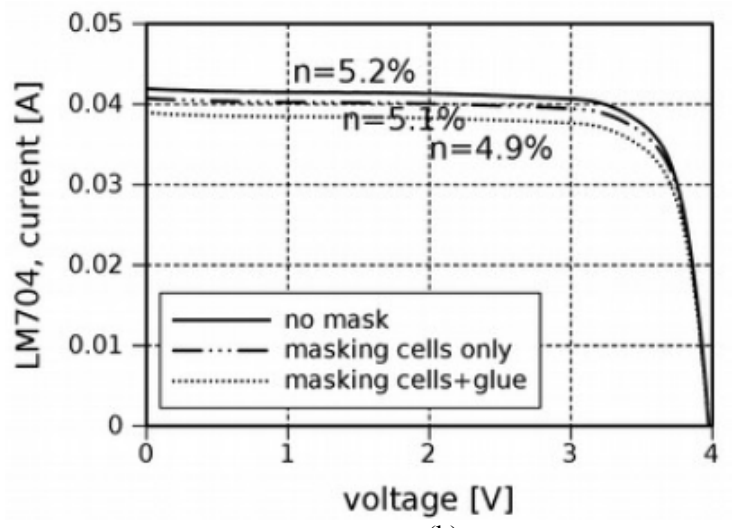

(b)

Fig. 6. LM704: (a) aperture area measurements performed at ESTI, highlighting the differences between the matrix area (2501.8 \pm 0.7 $\mathrm{mm}^{2}$, back dotted line), the matrix area plus the glue layer $\left(2536.3 \pm 0.7 \mathrm{~mm}^{2}\right.$, red dashed line) and the entire device area, including cell thickness; (b) comparison of module efficiencies calculated with the three different area definitions

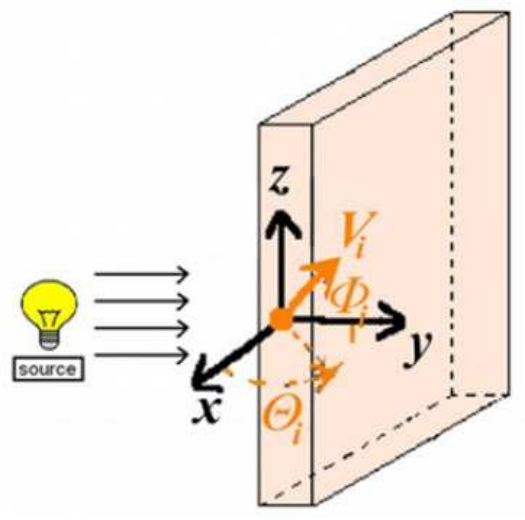

(a)

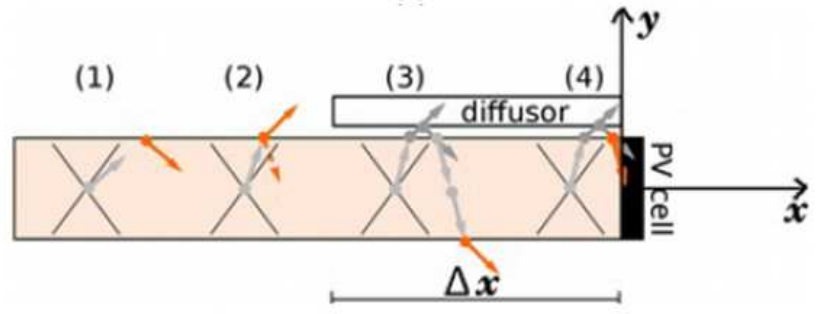

(b)

Fig. 7. Monte Carlo ray-trace modelling. (a) The coordinate system of reference; (b) Four possible events: Total internal reflection (1), refraction or Fresnel reflection (2), light diffused and lost (3), light diffused and collected (4)

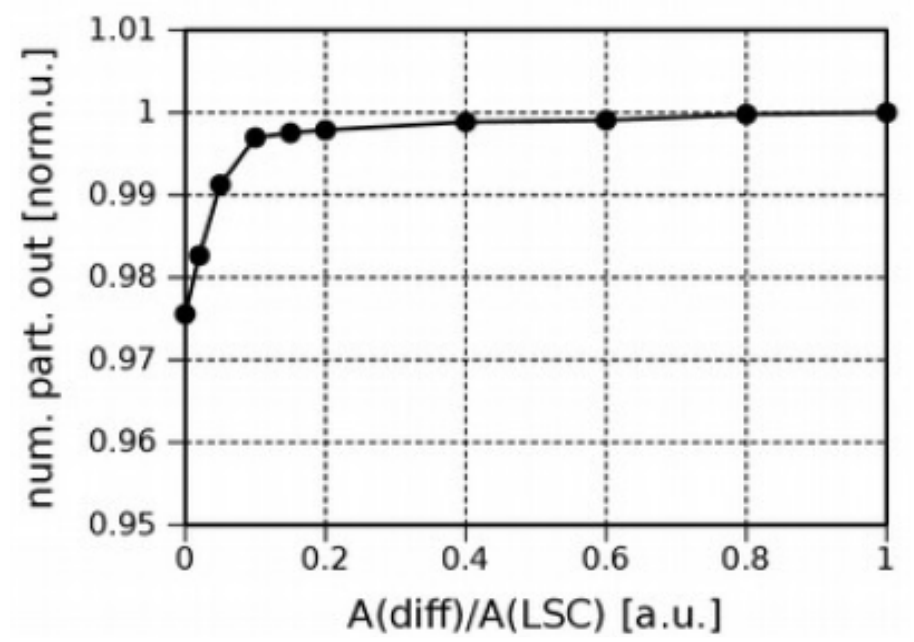

Fig. 8. EQE as a function of the diffuser area (Monte Carlo raytracing method): percentage of photons collected as a function of the diffuser area 


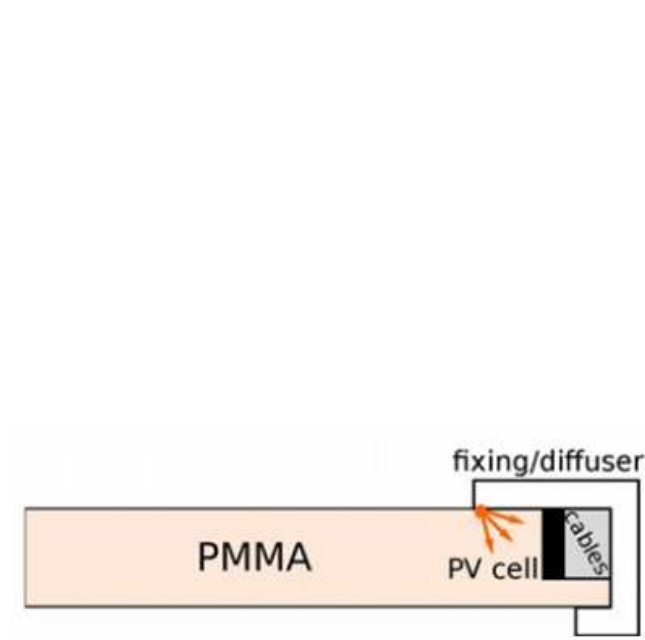

(a)

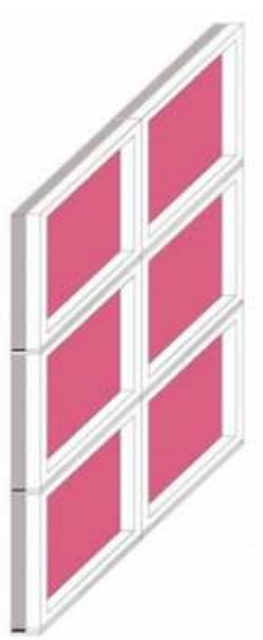

(b)

Fig. 9. Possible LSC design as smart windows: (a) The single LSC element (b) an array of LSCs (Pravettoni et al., 2009b)

The backside diffuser has been modelled by randomly diffusing back into the LSC matrix each particle exiting the $y>0$ interface if $-\Delta x \leq x \leq 0$. The size $\Delta x$ of the diffuser can be varied between 0 and the size of the tested LSC.

\section{Simulation Results}

Figure $7 \mathrm{~b}$ shows the four possible events modelled: (1) Light is total internal reflected; (2) light is refracted or Fresnel reflected; (3) light is reflected back into the matrix's escape cone and then is refracted (or Fresnel reflected) back from $y<0$ (secondary absorption is neglected); (4) light reflected back by the diffuser intercepts the index matched PV cell at the $x=0$ interface and is collected.

The code counts the fraction of particles collected at $x=0$ and those lost form the $y>0$ and $y<0$ interfaces.

Figure 8 shows the total number of collected particles in normalized units as a function of the ratio of area covered by the diffuser.

According to the basic ray-tracing approach, less than $3 \%$ more particles are collected by the diffuser and this contribution is almost totally due to the presence of the diffuser at the edges where PV cells are placed. This result gives qualitative confirmation of the results shown in the previous sections.

\section{Discussion}

Both experimental and computational results presented in the previous sections help to investigate on the impact of a backside diffusive reflector to the performance of a LSC of the given size.

The measured EQE of LSCs with different configurations of PV cells and diffusers showed that the impact of the latter can be twofold. As to light at wavelengths where luminescent species absorb, a diffusive reflector is shown to increase the absorption probability, by increasing the optical path of this fraction of light: Essentially, light that has not been absorbed while passing once through the LSC, has an additional chance after diffusion. As a matter of fact, at those wavelengths EQE seems to increase almost linearly with the diffuser area.

On the other hand, light at longer wavelengths than the luminescent cutoff is much more largely affected by the presence of a diffuser. The experimental results show also that the EQE at those wavelengths is more than doubled even when the diffuser is placed only close to the PV cell and not necessarily covering the entire backside.

The latter experimental result has been confirmed by the ray-tracing code. Based on the simplest classical optics approach, the diffuser may recycle the lost photons back to the interface: From that basic point of view, almost only the fraction of photons lost close to the edge where the PV cell is placed can be diffused into the cell and collected. All other recycled photons are lost from the opposite side of the LSC: Only particles diffused far from the PV cell edge at very wide angles may reach the cell. In the reality, such particles are most probably scattered and lost before reaching the cell.

Even if results shown in these paper may depend on the geometry of the LSC, the positive impact of a backside diffuser at the edges of the LSC is a result of general validity, being based on the optical properties described. What has been shown then suggests that, in view of the application of LSCs as smart windows, a full backside diffuser may be replaced by a thin diffusive frame all along the edges where PV cells are located. As a result the functionality of the window will not be affected but the LSC performance will benefit (Pravettoni et al., 2009b). 
In all the possible reflections for an appropriate integration of LSCs in buildings, other important factors need to be considered in the future, such perfect thermal insulation, the quality and quantity of the natural light income and the possible psychological effects due to color, for example in the working environments. The mere of integration of backside diffusers in the window profile makes the solution presented in this document extremely versatile and particularly interesting in building projects, but should take into account the fixing system, a perfect indoor ventilation with natural air exchange to the benefit of building's energy efficiency, the indoor welfare and air quality. A possible optimization of this element in the window profile is shown in Fig. 9: The fixing element ensures both mechanical stability and thermal and wet insulation but it also avoids shadowing the active area of the PV cell.

\section{Conclusion}

The EQE of LSCs has been analysed with three different approaches: (a) With the experimental setup in use for concentional PV cells and minimodules at ESTI; (b) with the modified experimental setup at ICL, allowing point-like EQE measurements and (c) with a basic Monte Carlo ray-tracing method. Results highlighted the twofold impact of a backside diffuser to the performance of LSCs: It both increases the absorption probability of light at wavelengths where luminescent species absorb and recycles light at wavelengths where the luminescent species is transparent. The latter effect is shown to be particularly important at the edges of LSCs, as the ray-tracing approach confirms. Adding a thin diffusive frame along the edges where PV cells are placed may therefore result in an improvement of the LSC performance, while not affecting its functionality as a smart window.

\section{Acknowledgement}

The authors would like to gratefully acknowledge Lenneke H. Slooff (ECN, The Netherlands) and Andreas Buchtemann (Fraunhofer-IAP, Germany) for their kind cooperation in providing LSC samples to be tested, Daniel J. Farrell, Amanda J. Chatten, Keith W.J. Barnham (Imperial College London, UK) and Roberto Galleano (ESTI, European Commission) for their valuable help and comments.

\section{Author's Contributions}

Mauro Pravettoni: Conducted the entire research and develped the experimental tools and computational activity.

Cristina S. Polo Lòpez: Conducted the review on LSC applications on building integration.
Robert P. Kenny: Supervised the research programe and gave important feedback on the research result.

\section{Ethics}

This article is original and contains unpublished material. The corresponding author confirms that all of the other authors have read and approved the manuscript and no ethical issues involved.

\section{References}

Barnham, K.W.J., J.L. Marques, J. Hassard and P. O'Brien, 2000. Quantum-dot concentrator and thermodynamic model for the global redshift. Applied Phys. Lett., 76: 1197-1199.

DOI: $10.1063 / 1.125981$

Batchelder, J.S., A.H. Zewail and T. Cole, 1979. Luminescent solar concentrators. 1: Theory of operation and techniques for performance evaluation. Applied Opt., 18: 3090-3110. DOI: $10.1364 / A O .18 .003090$

Batchelder, J.S., A.H. Zewail and T. Cole, 1981. Luminescent solar concentrators. 2: Experimental and theoretical analysis of their possible efficiencies. Applied Opt., 20: 3733-3754. DOI: 10.1364/AO.20.003733

Bose, R., D.J. Farrell, A.J. Chatten, M. Pravettoni and A. Buchtemann et al., 2008. Luminescent solar concentrators: Nanorods and raytrace modeling. Proceedings of the 33th IEEE Photovoltaic Specialists Conference, May 11-16, IEEE Xplore Press, San Diego, CA, USA, pp: 1-5. DOI: 10.1109/PVSC.2008.4922450

Carrascosa, M., S. Unamuno and F. Agullo-Lopez, 1983. Monte Carlo simulation of the performance of PMMA luminescent solar collectors. Applied Opt., 22: 3236-3241. DOI: $10.1364 / A O .22 .003236$

Chatten, A.J., D. Farrell, C. Jermyn, P. Thomas and B.F. Buxton et al., 2005. Thermodynamic modelling of luminescent solar concentrators. Proceedings of the 31th IEEE Photovoltaic Specialists Conference, Jan. 3-7, IEEE Xplore Press, pp: 82-85. DOI: 10.1109/PVSC.2005.1488074

Currie, M.J., J.K. Mapel, T.D. Heidel, S. Goffri and M.A. Baldo, 2008. High-efficiency organic solar concentrators for photovoltaics. Science, 321: 226- 228. DOI: $10.1126 /$ science. 1158342

Debije, M.G., J.P. Teunissen, M.J. Kastelijn, P.P.C. Verbunt and C.W.M. Bastiaansen, 2009. The effect of a scattering layer on the edge output of a luminescent solar concentrator. Solar Energy Mater. Solar Cells, 93: 1345-1350.

DOI: $10.1016 /$ j.solmat.2009.02.013 
Debije, M.G. and P.P.C. Verbunt, 2011. Thirty years of luminescent solar concentrator research: Solar energy for the built environment. Adv. Energy Mater., 2: 12-35. DOI: 10.1002/aenm.201100554

Farrell, D.J. and M. Yoshida, 2012. Operating regimes for second generation luminescent solar concentrators. Prog. Photovolt: Res. Applied, 20: 93-99. DOI: 10.1002/pip.1096

Goetzberger, A. and W. Greubel, 1977. Solar energy conversion with fluorescent collectors. Applied Phys., 14: 123-129. DOI: 10.1007/BF00883080

Goldschmidt, J.C., M. Peters, A. Bosch, H. Helmers and F. Dimroth et al., 2009. Increasing the efficiency of fluorescent concentrator systems. Solar Energy Mater. Solar Cells, 93: 176-182. DOI: 10.1016/j.solmat.2008.09.048

Green, M.A., K. Emery, Y. Hishikawa, W. Warta and E.D. Dunlop, 2016. Solar cell efficiency tables (version 47). Prog. Photovolt: Res. Appl., 24: 3-11. DOI: $10.1002 /$ pip. 2728

Mullejans, H., W. Zaaiman and R. Galleano, 2009. Analysis and mitigation of measurement uncertainty in the traceability chain for the calibration of photovoltaic devices. Meas. Sci. Technol., 20: 075101-075101. DOI: $10.1088 / 0957-0233 / 20 / 7 / 075101$

Polo Lopez, C.S., L. Tenconi, F. Lo Castro, S. Brambillasca and F.J. Neila Gonzalez et al., 2012. Controlled environment test laboratory for comfort performance studies on facade-integrated BIPV. Proceedings of the 27th European Photovoltaic Solar Energy Conference and Exhibition, (ECE' 12), Frankfurt, pp: 4335-4339.

Pravettoni, M., R. Bose, K.W.J. Barnham, A.J. Chatten, R.P. Kenny, 2008. Classical behaviour of output light emitted by the edge of a luminescent solar concentrator. Proceedings of the 33th IEEE Photovoltaic Specialist Conference, May 11-16, IEEE Xplore Press, San Diego, CA, USA, pp: 1-5. DOI: 10.1109/PVSC.2008.4922806

Pravettoni, M., D.J. Farrell, A.J. Chatten, R. Bose and R.P. Kenny et al., 2009a. External quantum efficiency measurements of luminescent solar concentrators: A study of the impact of backside reflector size and shape. Proceedings of the 24th European Photovoltaic Solar Energy Conference, (PSEC’ 09), pp: 332-335.
Pravettoni, M., F. Pravettoni, A. Virtuani, R.P. Kenny and A.J. Chatten et al., 2009b. Outdoor characterization of luminescent solar concentrators and their possible architectural integration on a historically relevant site in Milan (Italy). Proceedings of the 34th IEEE Photovoltaic Specialists Conference, Jun. 7-12, IEEE Xplore Press, Philadelphia, PA., pp: 187-192. DOI: 10.1109/PVSC.2009.5411701

Rowan, B.C., L. Wilson and B.S. Richards, 2008. Advanced material concepts for luminescent solar concentrators. IEEE J. Selected Top. Quantum Electron., 14: 1312-1322. DOI: $10.1109 /$ JSTQE.2008.920282

Sidrach De Cardona, M., M. Carrascosa, F. Meseguer, F. Cusso and F. Jaque, 1985. Edge effect on luminescent solar concentrators. Sol. Cells, 15: 225-230. DOI: 10.1016/0379-6787(85)90079-1

Slooff, L.H., R. Kindeman, A.R. Burgers, A. Buchtemann and R. Danz et al., 2006. The luminescent concentrator illuminated. Proc. SPIE, 6197: 61970K-61970K. DOI: $10.1117 / 12.662503$

Soti, R., E. Farkas, M. Hilbert, Z. Farkas and I. Ketskemety, 1995. Investigation of influence of reflection in luminescent layers II. Experimental analysis. J. Lumin., 63: 251-258. DOI: $10.1016 / 0022-2313(94) 00083-O$

Van Sark, W.G.J.H.M., K.W.J. Barnham, L.H. Slooff, A.J. Chatten and A. Buchtemann et al., 2008. Luminescent solar concentrators-a review of recent results. Opt. Express, 16: 21773-21792. DOI: $10.1364 /$ OE. 16.021773

Weber, W.H. and J. Lambe, 1976. Luminescent greenhouse collector for solar radiation. Applied Opt., 15: 2299-2300. DOI: 10.1364/AO.15.002299

Yablonovitch, E., 1980. Thermodynamics of the fluorescent planar concentrator. J. Opt. Soc. Am., 70: $1362-1363$. 